\title{
Experiencia de embolización de la arteria renal previa a nefrectomía
}

\section{Experience of Renal Artery Embolization Prior to Nephrectomy}

\author{
Diego Alejandro Arboleda-Gómez ${ }^{1}$ Johan Sebastián Lopera-Valle ${ }^{2}$ Luisa Fernanda Rueda-Cárdenas ${ }^{3}$ \\ Juliana Andrea Vergara-Cadavid ${ }^{4}$ José Miguel Hidalgo-Oviedo ${ }^{5}$
}

\footnotetext{
${ }^{1}$ Radiólogo, Hospital Universitario San Vicente Fundación, Medellín, Colombia

2 Residente de Radiología, Universidad de Antioquia, Medellín, Colombia

3 Residente de Neurología Clínica, Universidad CES, Medellín, Colombia

${ }^{4}$ Médica, Universidad de Antioquia, Medellín, Colombia

${ }^{5}$ Radiólogo Intervencionista, Hospital Universitario San Vicente

Fundación, Medellín, Colombia
}

\author{
Address for correspondence Johan Sebastián Lopera Valle, \\ Universidad de Antioquia, Medellín, Colombia \\ (e-mail: loperavalle@hotmail.com).
}

Rev Argent Radiol 2020;84:3-8.

\section{Resumen}

\section{Palabras clave \\ - embolización terapéutica \\ - nefrectomía \\ - neoplasias renales \\ - arteria renal}

\section{Abstract}

Objetivo Describir la evolución perioperatoria de pacientes sometidos a nefrectomía con y sin embolización de la arteria renal (EAR) prequirúrgica, en un hospital de alta complejidad de Medellín, Colombia.

Materiales y Métodos Observacional descriptivo retrospectivo; se incluyeron pacientes de 18-90 años con diagnóstico de tumor renal, sometidos a nefrectomía con y sin EAR prequirúrgica. Las variables cualitativas se expresaron por medio de frecuencias y proporciones, y las cuantitativas mediante medidas de tendencia central y dispersión.

Resultados Se incluyeron 71 pacientes con una media de edad de 58,1 (DE: 10,6) años, 41 eran mujeres y el $69 \%$ tenía diagnóstico de carcinoma de células claras. La media del volumen de sangrado intraoperatorio fue de 540,8 cc, y $19,7 \%$ requirió transfusión. El tiempo quirúrgico promedio fue de 2,6 horas y el $38 \%$ presentó alguna complicación, con una mortalidad total del $4,2 \%$. Al observar comparativamente los pacientes con EAR ( 15 pacientes) versus aquellos sin ella (56 pacientes), se identificó un mayor volumen de sangrado intraoperatorio y la necesidad de transfusión en los primeros.

Conclusión Los pacientes sometidos a EAR presentaron un mayor volumen de sangrado, mayor frecuencia de transfusión y complicaciones postoperatorias, siendo necesario un consenso sobre su real pertinencia terapéutica.

Aim To describe the perioperative outcomes of patients undergoing nephrectomy with and without preoperative Renal Artery Embolization (RAE) in a high-complexity hospital in Medellín, Colombia.

Materials and Methods Retrospective, descriptive and observational study; 18-90 years old patients with renal tumor diagnosis, submitted to nephrectomy with and without preoperative RAE were included. Qualitative variables were expressed by measures of frequencies and proportions, and quantitative variables were expressed by measures of central tendency and dispersion. received January 9, 2019 accepted July 17, 2019
DOI https://doi.org/ $10.1055 / \mathrm{s}-0039-3402487$ ISSN 1852-9992.
Copyright (c) 2020, Sociedad Argentina de Radiología. Publicado por Thieme Revinter Publicações Ltda., Rio de Janeiro, Brazil. Todos los derechos reservados.

\section{License terms}

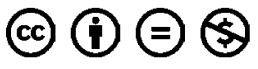


Keywords

- therapeutic embolization

- nephrectomy

- kidney neoplasms

- renal artery
Results 71 patients with a media age of 58,1 (SD: 10,6) years were included, 41 were women and $69 \%$ were diagnosed with clear cell carcinoma. The blood loss volume media was $540.8 \mathrm{cc}$, and $19.7 \%$ required transfusion. The operative time media was 2.6 hours and $38 \%$ had any complication, with a total mortality of $4.2 \%$. Comparatively observing patients with RAE (15 patients) versus patients without RAE (56 patients), a higher intraoperative blood loss and transfusion requirements were identified in the first ones.

Conclusion Patients submitted to RAE presented greater volume of bleeding, greater frequency of transfusion and post-operative complications, requiring a consensus on its real therapeutic relevance.

\section{Introducción}

La tasa de supervivencia a 5 años para el cáncer de riñón ha aumentado con el tiempo, tanto para la enfermedad localizada (de 88,4\% en el período 1992-1995 al 91,8\% entre 2004-2010) como para la enfermedad avanzada (de 7,3\% durante 19921995 al 12,3\% entre 2004-2010), atribuible probablemente a los recientes avances en alternativas mínimamente invasivas. ${ }^{1}$ Así pues, la embolización de la arteria renal (EAR), descrita en 1969 por Lalli y Peterson, indicada inicialmente en el tratamiento sintomático de la hematuria y en la paliación del cáncer metastásico renal, ${ }^{2}$ fue extendida en la década de 1970 y el interés inicial en la técnica dio lugar a múltiples series publicadas en 1980 y $1990 .^{3}$

Actualmente, las indicaciones de la EAR para el manejo de masas renales incluyen el tratamiento adyuvante preoperatorio de nefrectomía por masas primarias, paliación de síntomas relacionados con carcinoma de células renales avanzado y el tratamiento primario del angiolipoma renal. ${ }^{4}$ Dentro de los beneficios que justifican su uso se encuentran la disminución de la pérdida de sangre perioperatoria, la creación de un plano de tejido de edema facilitador de la disección, así como la reducción de la masa tumoral y la extensión del trombo vascular cuando está presente. ${ }^{5-7}$

Si bien la EAR pre nefrectomía se ha descrito en la literatura como un procedimiento seguro con potenciales beneficios perioperatorios, a la fecha no hay consenso que incentive su uso rutinario. ${ }^{6-8}$ Es de anotar que en nuestro medio ha entrado en desuso la EAR en el abordaje de tumores renales, probablemente por la discrepancia hallada en algunas series y el debate consecuente sobre su impacto real en esos desenlaces clínicos. El objetivo del presente estudio es describir la evolución perioperatoria de pacientes sometidos a nefrectomía con y sin EAR prequirúrgica, y atendidos en nuestra institución entre 2009 y 2016.

\section{Materiales y métodos}

Se realizó un estudio de tipo observacional descriptivo retrospectivo. Se incluyó a la totalidad de los pacientes de entre 18 y 90 años con diagnóstico tomográfico e histológico de tumor renal, a quienes se les realizó nefrectomía radical con y sin embolización completa prequirúrgica de la arteria renal en un hospital de alta complejidad de la ciudad de
Medellín, Colombia, entre los años 2009 y 2016. La indicación de EAR fue considerada como tratamiento adyuvante pre nefrectomía por masa renal primaria y no se consideró ningún criterio de exclusión.

Se empleó la técnica de embolización percutánea transcatéter por acceso arterial femoral ecoguiado, a través de introductor vascular $(5 \mathrm{Fr} \times 11 \mathrm{~cm})$ con técnica Seldinger y set de micropunción, utilizando guía hidrofílica $(0,035$ " $\mathrm{x}$ $150 \mathrm{~cm}$ ) y catéter tipo cobra (5Fr). De esa manera, se logró la oclusión mecánica del territorio vascular en cuestión (embolización renal completa) con 2-3 viales de micropartículas de alcohol polivinílico (PVA) de 300-500 o 700-1000 micrones (-Fig. 1). De igual forma, se utilizó medio de contraste yodado hidrosoluble no iónico de $300 \mathrm{mg} / \mathrm{ml}$ y un angiógrafo Philips Allura Xper FD10.

La nefrectomía radical fue realizada por el mismo grupo de cirujanos de la institución y fueron ellos quienes consignaron variables intraoperatorias como volumen de sangrado en el respectivo parte quirúrgico.

Se describieron características sociodemográficas, clínicas y quirúrgicas, haciendo uso de un formulario de recolección de datos diseñado por los investigadores para tal fin. La información de interés extraída de las historias clínicas fue consignada en los formularios y almacenada en una base de datos diseñada previamente por el equipo investigador.

El plan de análisis se realizó con base a los objetivos propuestos. Las variables cualitativas se expresaron por medio de frecuencias y proporciones y las cuantitativas se describieron mediante media y desviación estándar (DE), o mediana (Me) y rango intercuartil (RIQ) según su distribución. Los datos recolectados fueron analizados con el paquete estadístico SPSS versión 20.0.

La investigación obtuvo el aval del Comité de Ética de la institución para su ejecución. Además, se realizó con base en los principios éticos para la investigación, ciñéndose a la Declaración de Helsinki y resolución 008430 de 1993 del Ministerio de Salud de Colombia.

\section{Resultados}

En total, se incluyeron 71 pacientes con una media de edad de 58,1 años (DE: 10,6), de los cuales 41 eran mujeres (57,7\%), y solo a 15 de ellos se les realizó EAR previa nefrectomía en el periodo comprendido en el presente estudio, con un tiempo 

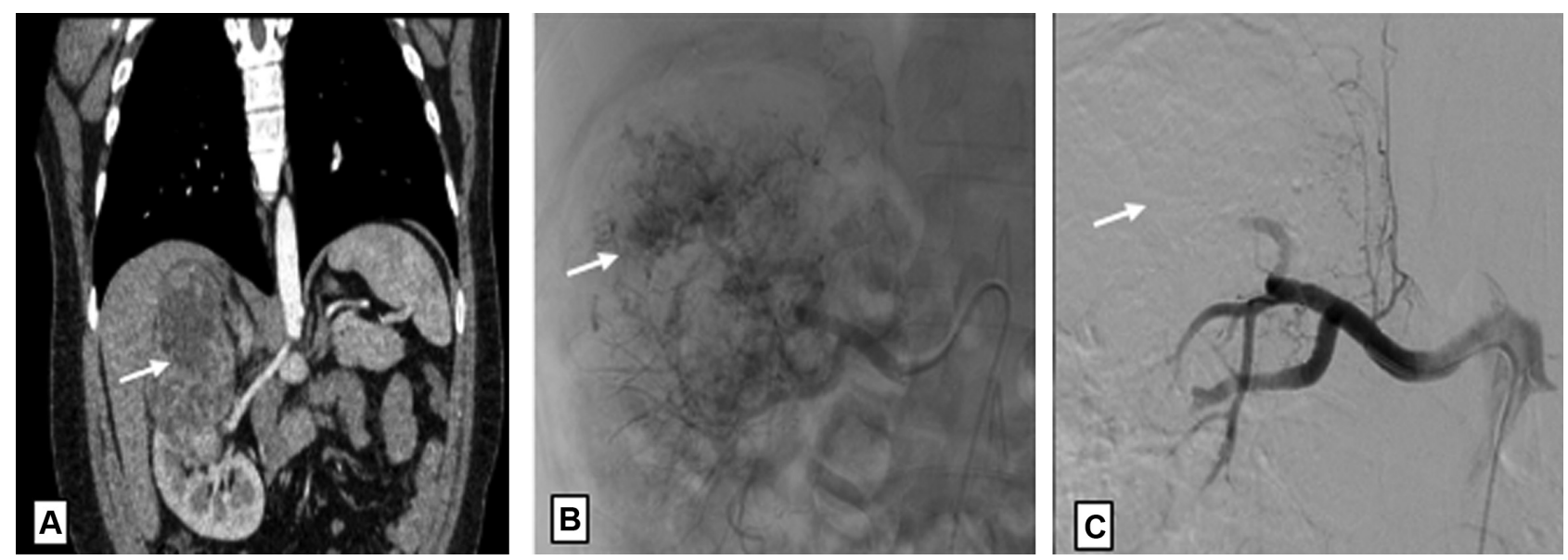

Fig. 1 Reconstrucción coronal de tomografía en fase arterial (A), así como imágenes pre (B) y post EAR (C) en paciente con carcinoma renal de células claras del polo superior derecho (flecha en A), empleando técnica de embolización percutánea transcatéter por acceso femoral con micropartículas de PVA. Angiográficamente, la lesión tumoral presenta irrigación arterial aumentada y neoformada (flecha en B). El control post embolización demuestra resultado satisfactorio con oclusión del territorio vascular de interés y ausencia de contraste intraparenquimatoso (flecha en C).

no mayor a 72 horas entre la embolización y la intervención quirúrgica. En la - Tabla 1 se detallan las características basales de los pacientes, con presencia de mayor proporción de carcinoma de células claras en los pacientes no sometidos a EAR prequirúrgica. La totalidad de los pacientes tenían recuento plaquetario mayor de $100.000 /$ $\mathrm{ml}$ e INR menor de 1,5 antes de ser llevados a cirugía.

Con respecto a las características oncológicas de los 71 pacientes en total, el $69 \%(n=49)$ tenían diagnóstico histológico de carcinoma de células claras, en más de la mitad (63,4\%) el tumor estaba limitado al riñón (T1 y T2). Solo 12 pacientes tenían diseminación confirmada a ganglios linfáticos regionales (N1), y 15 presentaban compromiso metastásico (M1) (-Tabla 2).

Tabla 1 Características basales de los pacientes sometidos a nefrectomía con y sin EAR prequirúrgica

\begin{tabular}{|c|c|c|}
\hline Variable & $\begin{array}{l}\text { CON EAR } \\
(n=15)\end{array}$ & $\begin{array}{l}\text { SIN EAR } \\
(n=56)\end{array}$ \\
\hline $\operatorname{Edad}[X(D E)]$ & $58,3(10,4)$ & $59,2(9,6)$ \\
\hline Sexo femenino [\% (n)] & $73,3(11)$ & $53,5(30)$ \\
\hline \multicolumn{3}{|l|}{ Tipo de Tumor Renal } \\
\hline $\begin{array}{l}\text { Carcinoma de células } \\
\text { claras [\% (n)] }\end{array}$ & $33,3(5)$ & $78,5(44)$ \\
\hline Carcinoma cromófobo [\% (n)] & $13,3(2)$ & $8,9(5)$ \\
\hline $\begin{array}{l}\text { Tumor de túbulos } \\
\text { colectores [\% (n)] }\end{array}$ & $6,6(1)$ & $1,7(1)$ \\
\hline Tumor sarcomatoide [\% (n)] & $6,6(1)$ & $1,7(1)$ \\
\hline Angiomiolipoma [\% (n)] & $6,6(1)$ & $1,7(1)$ \\
\hline Otros [\% (n)] & $20(3)$ & $10,7(6)$ \\
\hline \multicolumn{3}{|l|}{ Estadio Tumoral } \\
\hline Estadio T3-4 [\% (n)] & $53,3(8)$ & $32,1(18)$ \\
\hline Estadio N2-3 [\% (n)] & $26,6(4)$ & $14,2(8)$ \\
\hline Estadio M1 [\% (n)] & $33,3(5)$ & $17,8(10)$ \\
\hline
\end{tabular}

Abreviaturas: DE, Desviación estándar; X, Media.
En la -Tabla 3 se observan las características perioperatorias de los pacientes, con una media de seguimiento postquirúrgico de 11 días (DE: 2,7). La media del volumen de sangrado intraoperatorio fue de $540,8 \mathrm{cc}$, y el $19,7 \%(n=14)$ requirió transfusión de hemoderivados con una media de 2,7 unidades de glóbulos rojos. El tiempo quirúrgico promedio fue de 2,6 horas y en el $38 \%(n=27)$ de los pacientes se documentó alguna complicación postoperatoria, con una mortalidad atribuible al procedimiento quirúrgico realizado de tres pacientes $(4,2 \%)$ en el grupo sin EAR.

Tabla 2 Características oncológicas de los 71 pacientes

\begin{tabular}{|l|l|}
\hline Tipo de tumor renal & $\%(\mathbf{n})$ \\
\hline Carcinoma de células claras & $69(49)$ \\
\hline Carcinoma cromófobo & $9,9(7)$ \\
\hline Tumor de túbulos colectores & $2,8(2)$ \\
\hline Tumor sarcomatoide & $2,8(2)$ \\
\hline Angiomiolipoma & $2,8(2)$ \\
\hline Otro & $12,7(9)$ \\
\hline Estadio tumoral & $\%(\mathbf{n})$ \\
\hline Tamaño & \multicolumn{2}{|l|}{} \\
\hline T1 & $43,7(31)$ \\
\hline T2 & $19,7(14)$ \\
\hline T3 & $26,8(19)$ \\
\hline T4 & $9,9(7)$ \\
\hline Nódulo/Ganglio & $59,2(42)$ \\
\hline Nx & $23,9(17)$ \\
\hline N0 & $16,9(12)$ \\
\hline N1 & $25,4(18)$ \\
\hline Metástasis & $53,5(38)$ \\
\hline Mx & $21,1(15)$ \\
\hline M0 & \\
\hline M1 &
\end{tabular}


Tabla 3 Variables perioperatorias de los pacientes sometidos a nefrectomía con y sin EAR prequirúrgica

\begin{tabular}{|l|l|l|l|}
\hline Variable & $\begin{array}{l}\text { Todos los } \\
\text { pacientes } \\
(\boldsymbol{n}=\mathbf{7 1})\end{array}$ & $\begin{array}{l}\text { CON EAR } \\
(\boldsymbol{n}=\mathbf{1 5})\end{array}$ & $\begin{array}{l}\text { SIN EAR } \\
(\boldsymbol{n}=\mathbf{5 6})\end{array}$ \\
\hline $\begin{array}{l}\text { Sangrado } \\
\text { intraoperatorio } \\
(\mathrm{cc}) \text { [X (DE)] }\end{array}$ & $\begin{array}{l}540,8 \\
(729,2)\end{array}$ & $\begin{array}{l}896 \\
(729)\end{array}$ & $\begin{array}{l}445,7 \\
(705,6)\end{array}$ \\
\hline $\begin{array}{l}\text { Necesidad de } \\
\text { transfusión [\% (n)] }\end{array}$ & $19,7(14)$ & $40(6)$ & $14,2(8)$ \\
\hline $\begin{array}{l}\text { Numero de } \\
\text { UGRE [X (DE)] }\end{array}$ & $2,7(1)$ & $2,83(0.9)$ & $2,63(1.1)$ \\
\hline $\begin{array}{l}\text { Tiempo quirúrgico } \\
\text { (horas) [X (DE)] }\end{array}$ & $2,6(1,1)$ & $2,9(1.4)$ & $2,6(1)$ \\
\hline $\begin{array}{l}\text { Complicaciones } \\
\text { POP [\% (n)] }\end{array}$ & $38(27)$ & $40(6)$ & $29,6(21)$ \\
\hline $\begin{array}{l}\text { Mortalidad } \\
\text { POP [\% (n)] }\end{array}$ & $4,2(3)$ & $0(0)$ & $5,3(3)$ \\
\hline
\end{tabular}

Abreviaturas: DE, Desviación estándar; POP, Posoperatorio; UGRE, Unidad de glóbulos rojos empaquetados; X, Media.

Con respecto a las complicaciones postoperatorias de los 71 pacientes sometidos a nefrectomía, la más frecuente fue insuficiencia renal aguda en seis pacientes, seguido por íleo y dolor lumbar en el $7 \%$ cada una $(n=5)$. Cabe aclarar que ninguno de los pacientes sometidos a EAR presentó hematuria macroscópica o anemia secundaria al procedimiento.

Al observar las variables periperatorias, se identifica mayor volumen de sangrado intraoperatorio, mayor necesidad de transfusión ( - Tabla 3) y mayor dolor lumbar postoperatorio (-Tabla 4) en aquellos pacientes sometidos a EAR.

\section{Discusión}

En los Estados Unidos, el carcinoma de células renales constituye aproximadamente el 3,9\% de los nuevos casos de cáncer, con una edad media de 64 años al momento del diagnóstico y una relación hombre:mujer de 2:1. ${ }^{1}$ Cabe recordar que la media de edad de los pacientes incluidos en

Tabla 4 Complicaciones de los pacientes sometidos a nefrectomía con y sin EAR prequirúrgica

\begin{tabular}{|l|l|l|}
\hline Variable & $\begin{array}{l}\text { CON EAR } \\
(\boldsymbol{n}=\mathbf{1 5})\end{array}$ & $\begin{array}{l}\text { SIN EAR } \\
(\boldsymbol{n}=\mathbf{5 6})\end{array}$ \\
\hline HTA [\% (n)] & $6,6(1)$ & $3,5(2)$ \\
\hline Dolor lumbar [\% (n)] & $20(3)$ & $3,5(2)$ \\
\hline Fiebre [\% (n)] & $0(0)$ & $5,3(3)$ \\
\hline íleo [\% (n)] & $6,6(1)$ & $7,1(4)$ \\
\hline ISO [\% (n)] & $0(0)$ & $7,1(4)$ \\
\hline IRA [\% (n)] & $0(0)$ & $10,7(6)$ \\
\hline Otros [\% (n)] & $6,6(1)$ & $8,9(5)$ \\
\hline
\end{tabular}

Abreviaturas: HTA, Hipertensión arterial; IRA, Injuria renal aguda; ISO, Infección del sitio operatorio.

Otros: fístulas reno cutáneas, colecciones perirenales, lesiones vasculares y neumonía. este estudio fue 58,1 años, siendo ligeramente mayor en mujeres (relación H:M de 1,3:1). Según lo reportado en la literatura, el 50\% de los tumores se presentan en estadioT1-T2, ${ }^{9}$ lo cual resulta similar a los hallazgos presentados previamente, en los cuales cerca del 60\% se encontraban en dicho estadiaje.

Las opiniones sobre el papel de la EAR preoperatoria en el manejo de pacientes con patología tumoral renal aún son controvertidas, ${ }^{5}$ sin poder hallar en la literatura un consenso sobre los beneficios y morbilidad asociados con el procedimiento. ${ }^{9-11}$ A pesar de que se ha descrito la facilitación de la nefrectomía a través de la disminución de la pérdida de sangre, la facilidad de disección secundaria al edema en los planos tisulares y la disminución de los tiempos quirúrgicos con la EAR, 2,12 en el presente estudio se observó tendencia a mayor volumen de sangrado intraoperatorio y necesidad de transfusión en aquellos pacientes sometidos a embolización previa a la nefrectomía.

Los resultados previamente expuestos resultan discordantes con los reportados por autores como Maksimov y col., ${ }^{13}$ quienes describieron su experiencia en la realización de embolización superselectiva con balón de ramas segmentarias de la arteria renal previa nefrectomía parcial por neoplasia renal. De esa forma, observaron una disminución promedio en el sangrado operatorio y del tiempo quirúrgico. Por su parte, Yeast y col. $^{14}$ evaluaron el sangrado intraoperatorio y la tasa de transfusión de pacientes sometidos a nefrectomía de transplante con (grupo 1) y sin (grupo 2) embolización preoperatoria. La media de pérdida sanguínea para los pacientes embolizados fue de 143,9cc en comparación con $621,4 c c$ en el grupo $2(p=0,041)$. Por último, Ángeles y col. ${ }^{15}$ reportaron en México menor sangrado transoperatorio y mayor sobrevida en 23 pacientes sometidos a embolización prenefrectomía en comparación con 98 pacientes no embolizados.

Por otro lado, y de forma similar con lo observado en el presente estudio, May y col. ${ }^{16}$ no encontraron grandes diferencias en mortalidad ni complicaciones quirúrgicas en el estudio realizado entre 1992 y 2006 en 834 pacientes, tras comparar 227 pacientes sometidos a nefrectomía con EAR preoperatoria (grupo 1), versus 607 pacientes sin esta (grupo 2). Observaron, además, mayor necesidad de transfusión de sangre en el grupo 1 vs grupo 2 ( $61 \%$ vs $24 \%, p<0,01$ ), lo cual resulta similar a los hallazgos descritos en nuestro estudio. Subramanian y col., ${ }^{17}$ por su parte, concluyeron en 2009 que la embolización preoperatoria de la arteria renal en 135 pacientes sometidos a nefrectomía radical no proporciona ningún beneficio medible, asociándose por el contrario con mayor media de unidades transfusionales, mayor tiempo operatorio, mayores complicaciones perioperatorias y mayor mortalidad.

La EAR es considerada un procedimiento seguro y de baja morbilidad. ${ }^{18,19}$ Las complicaciones descritas incluyen infección del sitio operatorio, abscesos retroperitoneales, eventos cardiopulmonares, hemorragia secundaria, transfusión sanguínea, trombosis, apoplejía cerebral, íleo, síndrome postembolización y hematomas inguinales, entre otras. Las complicaciones más graves afectan entre el $2 \%$ y el $10 \%$ de los pacientes, con una mortalidad del 3,3\%. La más común suele ser el síndrome de postembolización, caracterizado por dolor 
lumbar, fiebre y náuseas, el cual se ha reportado hasta en $2 / 3$ de los pacientes sometidos a EAR. ${ }^{20,21}$

En el presente estudio, la tasa general de complicaciones, con y sin EAR prenefrectomía, fue alrededor del 38\% $(n=27)$, sin la presencia de casos de mortalidad asociados a la realización de la embolización. Zargar y col, ${ }^{8}$ por su parte, tras incluir 42 pacientes sometidos a EAR pre nefrectomía radical entre 2004 y 2011, de los cuales 7 de cada 10 tenían enfermedad avanzada (T3 y T4), documentaron alguna complicación operatoria en el 45,2\%.

Como se mencionó previamente en los resultados, se obtuvo una frecuencia del $8,5 \%$ para insuficiencia renal aguda (en comparación con el $2 \%$ reportado por Zielinski y col. $^{23}$ ), del $7 \%$ para íleo (9\% observado por Zielinski y col. ${ }^{23}$ ), del $7 \%$ para dolor lumbar (86\% documentado por Zielinski y col. $\left.^{23}\right)$, del 5,6\% para ISO (8,6\% reportado por May y col. $\left.{ }^{16}\right)$, del $4,2 \%$ para hipertensión arterial (75\% reportado por Zielinski y col. $^{23}$ y de un $51,5 \%$ por Pascual y col. ${ }^{24}$ ), así como $4,2 \%$ para fiebre (58\% reportado por Zielinski y col. ${ }^{23}$ ).

Dentro de las limitaciones del estudio, se destacan las derivadas de un diseño observacional, descriptivo retrospectivo, con una muestra reducida. Sin embargo, cabe considerar la baja frecuencia actual de realización de ese tipo de intervenciones y resulta compatible con las pequeñas series reportadas en 2016 por Maksimov y col. ${ }^{13}$ (11 pacientes), y por Yeast y col. ${ }^{14}$ (16 pacientes), y en 2015 por Thorlund y col. ${ }^{21}$ (15 pacientes).

De igual forma, resulta pertinente mencionar la posibilidad de sesgo de selección en el presente estudio, considerando la inexistencia de criterios claros para la realización de la EAR, cuya indicación fue considerada pertinente por el médico tratante como tratamiento adyuvante prenefrectomía por masa renal primaria. Por otro lado, un mayor porcentaje de pacientes a quienes se les realizó EAR se encontraban en un estadio más avanzado de la enfermedad, aspecto que pudo influir como variable de confusión en el tiempo quirúrgico, cantidad de sangrado y complicaciones.

Como conclusión, en nuestro estudio los pacientes sometidos a EAR presentaron un mayor volumen de sangrado, mayor frecuencia de transfusión y complicaciones post-operatorias, existiendo aun la controversia sobre el impacto real de la EAR en desenlaces clínicos y quirúrgicos relevantes. Si bien el diseño metodológico de este estudio impide la obtención de evidencia a partir de la cual se puedan generar pautas claras en el manejo quirúrgico de la población objeto de estudio, este es un primer paso en la epidemiología regional e incentiva la realización de estudios que evalúen el rendimiento de diferentes técnicas y agentes de embolización de acuerdo a su disponibilidad, accesibilidad y costos.

\section{Responsabilidades Éticas}

Protección de personas y animales. Los autores declaran que para esta investigación no se han realizado experimentos en seres humanos ni en animales.

Confidencialidad de los datos. Los autores declaran que han seguido los protocolos de su centro de trabajo sobre la publicación de datos de pacientes.
Derecho a la privacidad y consentimiento informado. Los autores declaran que en este artículo no aparecen datos de pacientes.

\section{Conflicto de Intereses}

Los autores declaran no tener ningún conflicto de interés.

\section{Bibliografía}

1 Motzer RJ, Jonasch E, Michaelson MD, Nandagopal L, Gore JL, George S, et al; National comprehensive cancer network. Kidney cancer, version 3.2015. J Natl Compr Canc Netw. 2015;13(02): 151-159

2 Provenza G, Sparagna A, Cunsolo GV, Tierno SM, Centanini F, Bellotti C, et al. Renal artery embolization in a gross kidney neoplasm. Case report. G Chir. 2013;34(9-10):263-266

3 Almgård LE, Slezak P. Treatment of renal adenocarcinoma by embolization: a follow-up of 38 cases. Eur Urol. 1977;3(05): 279-281

4 Li D, Pua BB, Madoff DC. Role of embolization in the treatment of renal masses. Semin Intervent Radiol. 2014;31(01):70-81

5 Madoff D, Verma R, Ahrar K. Embolotherapy for organ ablation. In: Golzarian J, Sun S, Sharafuddin M (eds.) Vascular Embolotherapy. A Comprehensive Approach. Heidelberg: Springer-Verlag; 2016: 201-220

6 Muller A, Rouvière 0 . Renal artery embolization-indications, technical approaches and outcomes. Nat Rev Nephrol. 2015;11 (05):288-301

7 Ramaswamy RS, Darcy MD. Arterial Embolization for the Treatment of Renal Masses and Traumatic Renal Injuries. Tech Vasc Interv Radiol. 2016;19(03):203-210

8 Zargar H, Addison B, McCall J, Bartlett A, Buckley B, Rice M. Renal artery embolization prior to nephrectomy for locally advanced renal cell carcinoma. ANZ J Surg. 2014;84(7-8):564-567

9 Martínez A, Sánchez LC, Calderón JE, Domínguez GL, Pedraza H, Ávila P. Comparación del tratamiento quirúrgico de los tumores renales con embolización y sin embolización de la arterial renal. Bol Coleg Mex Urol. 2010;25(02):82-90

10 Keller FS. Interventional radiology: new paradigms for the new millennium. J Vasc Interv Radiol. 2000;11(06):677-681

11 Rimon U, Duvdevani M, Garniek A, Golan G, Bensaid P, Ramon J, et al. Ethanol and polyvinyl alcohol mixture for transcatheter embolization of renal angiomyolipoma. AJR Am J Roentgenol. 2006;187(03):762-768

12 Qin C, Wang Y, Li P, Li P, Tao J, Shao P, et al. Super-Selective Artery Embolization before Laparoscopic Partial Nephrectomy in Treating Renal Angiomyolipoma. Urol Int. 2017;99(03): 277-282

13 Maksimov AV, Martov AG, Pavlov LP, Neustroev PA, Vinokurov RR. [Laparoscopic partial nephrectomy with superselective balloon embolization of renal artery]. Urologiia. 2017;1(01):31-36

14 Yeast C, Riley JM, Holyoak J, Ross G Jr, Weinstein S, Wakefield M. Use of preoperative embolization prior to Transplant nephrectomy. Int Braz J Urol. 2016;42(01):107-112

15 Ángeles A, Sánchez LC, Calderón JE, Domínguez G, Lujano H, Ávila P. Comparación del tratamiento quirúrgico de los tumores renales con embolización y sin embolización de la arterial renal. Bol Coleg Mex Urol. 2010;25:82-90

16 May M, Brookman-Amissah S, Pflanz S, Roigas J, Hoschke B, Kendel F. Pre-operative renal arterial embolisation does not provide survival benefit in patients with radical nephrectomy for renal cell carcinoma. Br J Radiol. 2009;82(981):724-731

17 Subramanian VS, Stephenson AJ, Goldfarb DA, Fergany AF, Novick AC, Krishnamurthi V. Utility of preoperative renal artery embolization for management of renal tumors with inferior vena caval thrombi. Urology. 2009;74(01):154-159 
18 Schwartz MJ, Smith EB, Trost DW, Vaughan ED Jr. Renal artery embolization: clinical indications and experience from over 100 cases. BJU Int. 2007;99(04):881-886

19 Ginat DT, Saad WE, Turba UC. Transcatheter renal artery embolization: clinical applications and techniques. Tech Vasc Interv Radiol. 2009;12(04):224-239

20 Guziński M, Kurcz J, Tupikowski K, Antosz E, Słowik P, Garcarek J. The Role of Transarterial Embolization in the Treatment of Renal Tumors. Adv Clin Exp Med. 2015;24(05):837-843

21 Thorlund MG, Wennevik GE, Andersen M, Andersen PE, Lund L. High success rate after arterial renal embolisation. Dan Med J. 2015;62(05):1-3
22 Gueglio G, Piana M, García R, Peralta O, Damia O. Lugar actual de la embolización transarterial en tumores renales. Rev Argent Urol. 2005;70(01):31-41

23 Zielinski H, Szmigielski S, Petrovich Z. Comparison of preoperative embolization followed by radical nephrectomy with radical nephrectomy alone for renal cell carcinoma. Am J Clin Oncol. 2000;23(01):6-12

24 Pascual A, Calleja J, Pesquera L, Martín S, Arreba E, Cortiñas JR. Indicaciones y complicaciones de la embolización renal: Análisis retrospectivo. Rev Chil Urol. 2014;79(01):24 